Вестник образования и развития науки Российской академии естественных наук, 2018, 22(1): 65-72 (C) С.И. Сороко, С.С. Бекшаев, 2018

S.I. Soroko, S.S. Bekshaev

\title{
NEW TECHNOLOGY OF CORRECTION OF NEURO- PSYCHIC STATES USING BIOFEEDBACK REGULATION OF POWER AND LOCALIZATION OF ELECTRIC DIPOLE OF EEG
}

Svyatoslav Soroko - Head of the Laboratory, Sechenov Institute of Evolutionary Physiology and Biochemistry of Russian Academy of Sciences, corresponding member of Russian Academy of Sciences, full member of Russian Academy of Natural Sciences, recepient of the State Prize of the USSR, Doctor of Medicine, professor, St. Petersburg; e-mail: soroko@iephb.ru.

Sergey Bekshaev - senior researcher, Sechenov Institute of Evolutionary Physiology and Biochemistry of Russian Academy of Sciences, PhD in Biology, mathematician, specialist in the field of Neurocybernetics, St. Petersburg; e-mail: mbeks47@yandex.ru.

We discuss the new approaches to the correction of functional disorders of the central nervous system using the adaptive bio-control of electrical activity of the brain (neurobiofeedback regulation method). We present the data concerning the use of power and localization of electric dipole sources as feedback signals for the first time. We show that the power decrease or moving the dominant focus of dipole activity from the deep and cortical brain structures that support the pathological state result in the disappearance of disorder symptoms and normalization of the functional state.

Keywords: brain; functional states; neurobiofeedback method; localization of electric dipole; correction of functional disorders.

С.И. Сороко, С.С. Бекшаев

\section{НОВАЯ ТЕХНОЛОГИЯ КОРРЕКЦИИ НЕРВНО- ПСИХИЧЕСКИХ СОСТОЯНИЙ С ПОМОЩЬЮ НБОС-РЕГУЛЯЦИИ МОЩНОСТИ И ЛОКАЛИЗАЦИИ ЭЛЕКТРИЧЕСКИХ ДИПОЛЬНЫХ ИСТОЧНИКОВ ЭЭГ}

\begin{abstract}
Святослав Иосифович Сороко - заведующий лабораторией Института эволюционной физиологии и биохимии им. И.М. Сеченова Российской академии наук, член-корреспондент РАН, действительный член РАЕН, лауреат Государственной премии СССР, доктор медицинских наук, профессор, г. СанктПетербург; e-mail: soroko@iephb.ru.

Сергей Степанович Бекшаев - старший научный сотрудник Института эволюционной физиологии и биохимии им. И.М. Сеченова Российской академии наук, кандидат биологических наук, математик, специалист в области нейрокибернетики, г. Санкт-Петербург; e-mail: mbeks47@yandex.ru.
\end{abstract}

В статье рассматриваются новые подходы к коррекции функциональных нарушений центральной нервной системы человека с помощью адаптивного биоуправления электрической активностью мозга (НБОС-регуляция). Впервые приводятся данные по использованию в качестве сигналов обратной связи мощности и локализации электрических дипольных источников. Показано, что подавление мощности или перемещение доминирующего очага дипольной активности из глубоких и корковых структур мозга, поддерживающих патологическое состояние, приводит к исчезновению симптоматики нарушений и нормализации функционального состояния.

Ключевые слова: мозг; функциональные состояния; нейробиоуправление с биологическими обратными связями; локализация электрических диполей; коррекция функциональных расстройств. 
Разработка технологий сознательного контроля неосознаваемых процессов в организме с помощью биологической обратной связи (далее - БОС), которые позволяют иногда более эффективно, чем лекарства, помочь в восстановлении нарушенных функций у больных после инсульта, гипертоникам, с мигренью и головной болью, астматикам, при тревожных и депрессивных расстройствах и т.д. - получает все большее практическое приложение в клинической медицине. В последние годы получены экспериментальные доказательства использования этого метода в дифференциальной диагностике ряда заболеваний, когда с помощью общепринятых методов это требует значительно больших усилий и не всегда удается.

Теоретические предпосылки современной психофизиологии «биологической обратной связи» (БОС) относятся к середине XX столетия. Практическое же использование определенных БОС методов и процедур началось 30-40 лет назад. Они используются не только как монотерапия, но также в комбинации с современной психотерапией, аутогенной тренировкой и гипнозом. БОС-технологии расширяют методы лечения, используемые врачами и медицинскими психологами. Существует тесное взаимодействие между психофизиологическими исследованиями и изучением эффектов и механизмов БОС. Методы адаптивного биоуправления могут с успехом использоваться не только для изучения механизмов регулирования физиологических функций, но и для направленной тренировки и мобилизации функциональных резервов организма с целью облегчения процессов адаптации и коррекции возможных дезадаптационных расстройств.

Наши исследования показали, что коррекция функционального состояния методами адаптивного биоуправления с БОС происходит не столько за счет абсолютных изменений регулируемого параметра, сколько за счет перестроек структуры внутрисистемных и межсистемных взаимоотношений. Это свидетельствует о том, что направленная произвольная мо- дификация физических параметров биоэлектрической активности (ее амплитудных и частотных характеристик, взаимосвязи компонентов и т.д.) одной физиологической системы вовлекает в ответ и сопрягает с ней другие системы, участвующие в поддержании общего гомеостаза, меняя тем самым функциональное состояние организма в целом $[9 ; 10]$.

Однако следует отметить, что выбор того или иного методического приема, его тактика должны определяться индивидуальными особенностями механизмов саморегуляции, характером возникших перестроек функций, эффективностью и возможностями коррекции состояния по тем или иным параметрам БОС. Для коррекции функциональных нарушений ЦНС до сих пор используется регуляция амплитудных или частотных параметров текущей ЭЭГ, т.е. суммарная биоэлектрическая активность мозга, регистрируемая с поверхности головы. В то же время является весьма заманчивым подход, при котором можно было бы ввести в систему обратной связи для направленной регуляции активность конкретных корковых или подкорковых структур мозга. В микроэлектродных экспериментах на животных показано, что введение в систему обратной связи активности отдельных нейронов или нейронных популяций позволяет направленно изменять не только их активность, но и обучать нейронные сети поддерживать то или иное функциональное состояние [10]. Однако использование этой методики на здоровом человеке невозможно, поскольку требует хирургического вмешательства с введением электродов непосредственно в мозг.

Начиная с 60-х годов, появляются работы, в которых ставится задача определения координат корковых и подкорковых структур головного мозга человека по потенциалам, регистрируемым электродами, расположенными на скальпе $[1 ; 3$; $13 ; 14]$, то есть по ЭЭГ (проблема локализации источников ЭЭГ). В постановках этих задач предполагается, что в каждый момент времени пространственная конфигурация биоэлектрического поля на коре головного мозга формируется электриче- 
ской активностью корковых и подкорковых структур и каждая из них вносит свой вклад в формирование этого поля $[4 ; 5 ; 6$; 7]. Очевидно, что некоторые из этих структур вносят наибольший вклад в формирование поля, они и называются доминирующими.

В такой постановке задачи определяются координаты доминирующего источника в каждый момент времени. Ясно, что координаты его не являются постоянными в силу динамического характера функционирования ЦНС.

Исследования в этой области обуславливались как теоретическими запросами (изучение механизмов работы мозга), так и потребностями медицины - поисками методов оценки состояния мозга и определения местоположения патологических очагов электрической активности (опухоли мозга, инсульты, эпилептические очаги и т.п.). В это же самое время развивалось направление нейровизуализации (neuroimaging), основанное на прогрессе в области техники физических измерений, химии и компьютерной технологии, а именно: ЯМР, ПЭТ, компьютерная томография [16].

Широкое внедрение этих методов в клиническую практику в 80-90-х годах принесло ощутимый прогресс в диагностику патологии мозга и отодвинуло на задний план дальнейшее развитие методов и исследований в области локализации источников по поверхностной ЭЭГ.

Перечисленные современные методы решают определенные задачи и имеют свои ограничения и недостатки: инвазивность, невозможность прослеживать быстрые (доли секунды) изменения активности мозга. Кроме того, стоимость их так велика, что не позволяет с нужной частотой проводить обследования. В случае же функциональных нарушений работы мозга томографические методы вообще неэффективны для определения местоположения очагов патологической активности, так как в данных ситуациях нет органических отклонений, а существует только динамическое во времени перераспределение уровня активности различных структур мозга и порядка их доминирова- ния. Эти ограничения не позволяют использовать информацию, получаемую с помощью указанных методов в реальном масштабе времени, который был бы сопоставим с характерными временными масштабами биоэлектрической активности головного мозга, а, следовательно, исключают возможность их применения в технологии БОС-регуляции состояний.

Пространственное разрешение, достигаемое в настоящее время при определении координат источников, порождающих ЭЭГ, составляет 0,1-0,4 см. Эти размеры соизмеримы с характерными размерами морфологии головного мозга.

Время, необходимое для оценки расстояния до «доминирующего источника», составляет, в зависимости от алгоритма обработки и производительности используемой ЭВМ, от 0,1 сек до нескольких секунд.

Пространственное расположение одного или нескольких доминирующих источников и изменение во времени уровня их биоэлектрической активности соответствует текущему функциональному состоянию головного мозга и одновременно определяет его (особенно в условиях патологии). В условиях патологии имеется преимущественно устойчивая локализация доминирующего источника в определенных областях мозга. Изменение биоэлектрической активности патологического очага приводит, как правило, к улучшению функционального состояния головного мозга и к нормализации его функций.

Афферентный одномодальный сигнал (например, звуковой), являющийся сигналом обратной связи, преобразуется центральной нервной системой в целый спектр ассоциированных с ним внутренних сигналов и процессов, которые находят свое отражение в текущем, пространственно распределенном по коре головного мозга полем биоэлектрических потенциалов. Указанные ассоциированные процессы приводят в действие компенсаторные механизмы головного мозга, которые, в свою очередь, активно используют резервные возможности ЦНС. Таким образом, головной мозг сам находит внутрен- 
ние способы нормализации нарушенных функций, используя информацию, поступающую через сенсорные каналы по цепи обратной связи [10].

Поскольку сигналы, поступающие по цепи обратной связи, физиологически адекватны организму (слуховые, зрительные, тактильные и т.п.), то они естественным образом включаются в функционирование ЦНС, не вызывая в ее работе отклонений, выводящих за пределы нормы.

В традиционных реализациях ЭЭГБОС сигналы обратной связи вырабатываются в зависимости от тех или иных изменений в ЭЭГ (наличие того или иного частотного компонента, его выраженности и т.д.), зарегистрированной в одном или максимум двух отведениях. Поэтому БОС, изменяя непосредственно текущую ЭЭГ, действует только опосредовано на активность подкорковых структур.

Однако если воспользоваться информацией о «доминирующих источниках» и включить ее в цепь, реализующую БОС, то можно воздействовать непосредственно на подкорковые структуры или образования, которые порождают патологическую активность.

Пространственное и временное разрешение методов «локализации источников» вполне позволяет использовать информацию о них в реальном масштабе времени.

Для реализации этого необходимо включить характеристики доминирующего источника в цепь биологической обратной связи.

Метод оценки локализации эквивалентных электрических дипольных источников (далее - ЭЭДИ) позволяет с помощью трехмерного компьютерного сечения мозга в трех плоскостях выявлять структуры мозга, где плотность ЭЭДИ оказывается наибольшей, прослеживать моменты появления и направление (вектор) движения этих диполей как внутри отдельных образований мозга, так и их перехода на другие подкорковые и корковые структуры.

В настоящее время имеется ряд компьютерных программ (в том числе и метод 3DLoc, входящий в пакет программ обработки ЭЭГ электроэнцефалографаанализатора ЭЭГА-21/26 комплекса «Энцефалан 131-03-10» (фирма «Медиком МТД», Таганрог, Россия), а также зарубежные коммерческие программы Biologic (Dipole 1.33), BESA (Brain electrical source analysis), BRAINLOC (Brain localization system), и др. Однако во всех этих программах для расчета локализации источников используются размеры модельной формы черепа, не позволяющие учитывать индивидуальные особенности конкретного человека, что вносит большие погрешности $[3 ; 11]$.

В своих исследованиях мы использовали программу 3DLocEEG, разработанную математиком, кандидатом биологических наук С.С. Бекшаевым [2].

Для того, чтобы читателям, незнакомым с данной технологией, были понятны полученные результаты, приведем краткое описание процедуры анализа и его отличий от имеющихся аналогов.

Совокупность электрических потенциалов, регистрируемых одновременно в различных пространственно разнесенных точках, расположенных на скальпе (т.е. собственно ЭЭГ), порождается многочисленными распределенными и сосредоточенными источниками тока, расположенными в мозге в силу протекающих там биоэлектрических процессов. Согласно классической теории поля [8], потенциал электрического поля вне области указанных источников (ЭЭГ) в точке с радиусвектором $R_{0}$, создаваемый указанными биоэлектрическими явлениями в точках с радиус-векторами $r_{i}$ (система координат выбрана внутри мозга), представляют в виде:

$$
\varphi=\sum e_{i} /\left(\left|R_{0}-r_{i}\right|\right)
$$

где $e_{i}-$ потенциалы, создаваемые упомянутыми выше источниками в мозге. Естественно, что $\left(R_{0}-r_{i}\right)$ является радиусвектором от $r_{i}$ до точки, в которой необходимо определить потенциал $\varphi\left(R_{0}\right)$. После разложения потенциала по степеням $\left(1 / R_{0}\right)$, получают бесконечный ряд: $\varphi\left(R_{0}\right)=\varphi(0)\left(R_{0}\right)+\varphi(1)\left(R_{0}\right)+(2)\left(R_{0}\right) \ldots$

Ряд (2) называют мультипольным разложением потенциала. Первый член 
ряда (2) представляет собой сумму всех зарядов, и для ЭЭГ можно полагать его равным нулю, поскольку в целом головной мозг электронейтрален. Второй член ряда называют дипольным потенциалом. Он определяется дипольным моментом (вектором, полученным при предельном сближении либо разноименных зарядов, либо источника и стока), направление которого совпадает с осью диполя, а длина равна расстоянию между разноименными зарядами из которых образован диполь. Третий член ряда - квадрупольный потенциал и т.д. Чем больше членов ряда (2) удерживают, тем точнее описание потенциала $\varphi$. Поскольку в (2) $\varphi(n)$ пропорционален $1 / R_{0}^{(n+1)}$, то на больших расстояниях $R_{0}$ наибольший вклад в общий потенциал вносит дипольная компонента, если она не равна нулю (в противном случае основную роль начинает играть квадрупольный потенциал). Если в (2) оставить только $\varphi(1)\left(R_{0}\right)$, то получим описание биопотенциального поля ЭЭГ в форме потенциалов дипольных источников. Биофизически диполь рассматривается как совокупность предельно близких, по сравнению с расстоянием до точки наблюдения, точечных источника и стока. Например, по мере распространения потенциала действия по волокну токи выходят из одной пространственной точки и входят в другую. Каждый диполь описывают его координатами и вектором его момента. Длина этого вектора пропорциональна величине дипольного тока. Если оценивать мощность дипольного источника, то она будет пропорциональна длине вектора дипольного момента, поэтому для удобства в дальнейшем будем величину вектора диполя называть «мощностью диполя».

В программе 3DLocEEG [2] для решения обратной задачи ЭЭГ используется модель подвижного эквивалентного электрического диполя в изотропном пространстве. Алгоритм, лежащий в основе разработанной компьютерной программы, основан на мультипольном разложении электрического поля, зарегистрированного с множественных (21) ЭЭГ электродов. Применен принцип минимизации вклада недипольных компонентов в пространственный профиль потенциального поля ЭЭГ, в данном случае минимизируется вклад квадрупольного члена. Получаемые формулы позволяют оценивать трехмерные координаты эквивалентных динамических диполей даже в реальном времени и избавиться от проблем сходимости к локальным минимумам в итерационных алгоритмах, применяемых в аналогичных программах.

Модель однородной среды выбрана для исключения параметров (проводимость в разных направлениях), плохо поддающихся измерению и контролю in vivo. Тем более известно, что результаты, полученные на однородных и на неоднородных многослойных моделях, отличаются незначительно.

Ключевой проблемой локализации является корректное сопоставление полученных координат источников реальным структурам и областям мозга. Эта проблема не может быть решена при использовании модельных форм черепа.

Из литературных источников известно, что неправильная постановка электродов (даже с разбросом в 1,0 см) дает до $20 \%$ ошибки в оценке координат эквивалентных электрических источников. Эти данные относятся к тем программам 3-х мерной локализации, в которых используется идеализированная форма черепа (сферическая, эллипсоида вращения и т.п.).

В программе 3DLocEEG этой проблемы не существует, поскольку трехмерные координаты ЭЭГ электродов определяются на основе измерений реальных межэлектродных расстояний и размеров черепа по его реперным точкам (в сагиттальном: inion-nasion; фронтальном: внешние ушные проходы, и вертикальном: вертекс базис направлениям), а также расстояний между электродами (всего 32 измерения). По этим данным вычисляются координаты регистрирующих электродов в трехмерном пространстве, которые затем используются для аппроксимации поверхности черепа.

Для каждого отсчета оцифрованной ЭЭГ в соответствии с указанным алго- 
ритмом вычисляются координаты эквивалентного электрического диполя. Потенциалы ЭЭГ непрерывно меняются, поэтому и точка с указанными координатами перемещается в пространстве. Траектория этой точки визуализируется и отображается на анатомические структуры головного мозга в соответствии с данными атласа Mai, Assheuer, Paxinos [15].

Точки отображаются на срезы мозга (сагиттальные, горизонтальные и фронтальные) толщиной в 1,0 см - всего 36 срезов. Срезы масштабируются по размерам черепа пациента в соответствии с рекомендациями атласа [17] и проведенными измерениями. Эти измерения используются в программе для триангуляции поверхности черепа и в численном интегрировании при решении обратной задачи ЭЭГ.

В используемой нами реализации программы частота оцифровки составляет 250 Гц, поэтому через каждые 4 мс получается новая точка с координатами. После получения всех точек на выбранном заранее временном отрезке ЭЭГ находятся точки с максимальной «мощностью диполя» («доминирующие источники»). Варьируя величиной порога (в процентах к максимальному), убирают из срезов точки с минимальной величиной «мощности», которые зачастую имеют шумовую природу вследствие невозможности абсолютно освободиться от артефактов различного характера при записи ЭЭГ.

Для получения информации об особенностях локализации эквивалентных дипольных источников (в дальнейшем просто диполей) в трехмерном пространстве головного мозга подсчитывается количество диполей (в определенном и фиксированном диапазоне «мощности диполя»), попадающих в тот или иной пространственный срез (толщиной 1,0 см) за определенный промежуток времени. Затем проводится нормировка количества диполей, содержащихся в каждом срезе, на общее количество зарегистрированных диполей. Таким образом, получаем информацию о проценте диполей, содержащихся в каждом срезе. Считаем, что, чем выше процент, тем выше активность в этом срезе. В программе предусмотрена возможность оценки количества диполей, попавших в интересующую нас область мозга, и подсчета их процента по отношению к общему количеству диполей в срезе (относительная плотность). Этот показатель призван отражать относительный уровень активности выбранной области.

Если в качестве параметра, по которому ведется управление, выбрать уровень снижения активности «патологического доминирующего источника» и при этом его координаты должны быть фиксированы, то в этом случае БОС будет направлена на подавление активности патологического очага (рис. 1).

Если в качестве параметра выбирать регуляцию расстояния до «патологического источника», то можно навязать активность структуре, располагающейся в другой пространственной области, - т.е. будет осуществляться перемещение доминирующего очага и размывание очага, поддерживающего патологическое состояние (рис. 2).

Данные, приведенные на рис. 1 и рис. 2 , показали, что пациент успешно освоил метод произвольной регуляции мощности и стереотаксической локализации дипольных источников и научился подавлять сложный моторный тик, включавший периодическое судорожное сокращение мышц лица и верхних конечностей (тик). После 10 сеансов НБОС-регуляции тик исчез полностью.

Интересным оказался тот факт, что предел БОС обучаемых сдвигов ЭЭГ у человека в состоянии нормы практически не выходит за его диапазон дисперсии, зарегистрированной в различных состояниях испытуемого. Это свидетельствует о том, что устойчивость механизмов саморегуляции мозга как единой системы поддерживается в норме и не допускает их выхода за определенный предел. Нарушение этих пределов является признаком дестабилизации нормальных межцентральных взаимоотношений и приводит к возникновению дезадаптационных расстройств или болезням регуляции [18].

Границы возможных биоуправляемых сдвигов параметров ЭЭГ зависят от их 
a

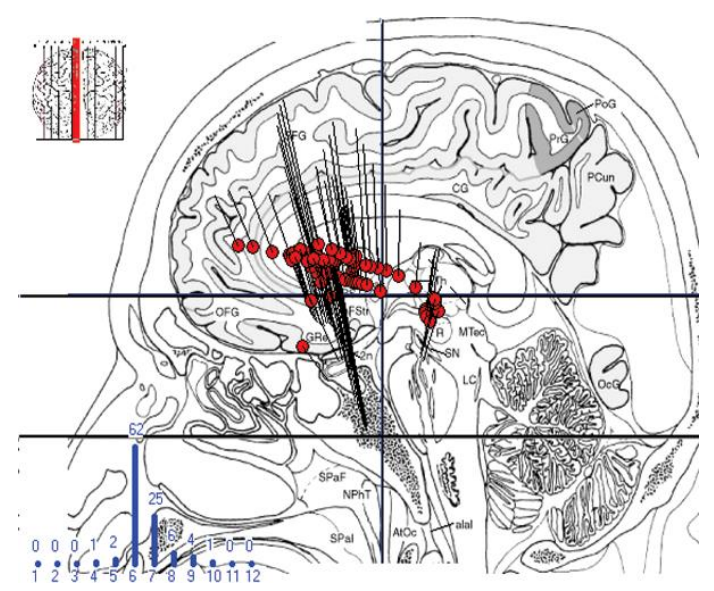

6

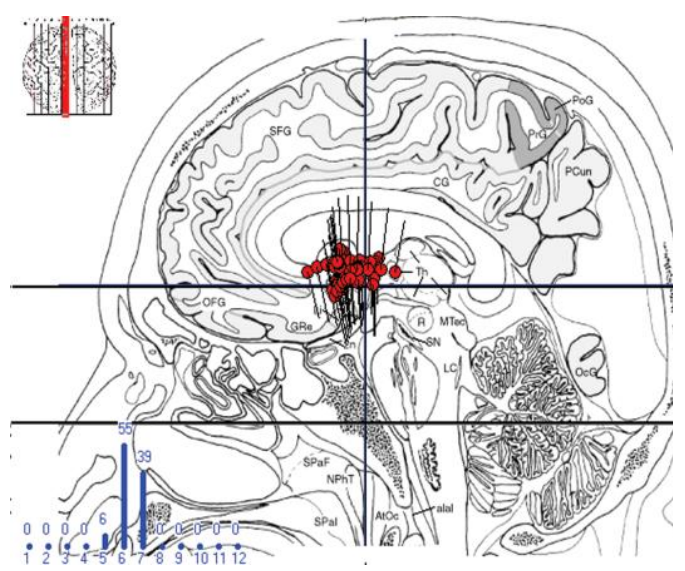

Рис. 1. Подавление активности глубоких структур мозга в сагиттальном разрезе левого полушария на уровне координаты 6, поддерживающих моторный тик, с помощью направленной регуляции мощности очага дипольных источников Примечание: а - до курса НБОС-регуляции, б - после НБОС-регуляции. На гистограммах, изображенных в левой нижней части рисунков, показано распределение количества ЭЭДИ по сагиттальным срезам в процентном отношении к общему числу зарегистрированных диполей (числа над столбиками). Номера срезов указаны под гистограммой (с 1 по 6 срез - левое полушарие, с 7 по 12 - правое полушарие).

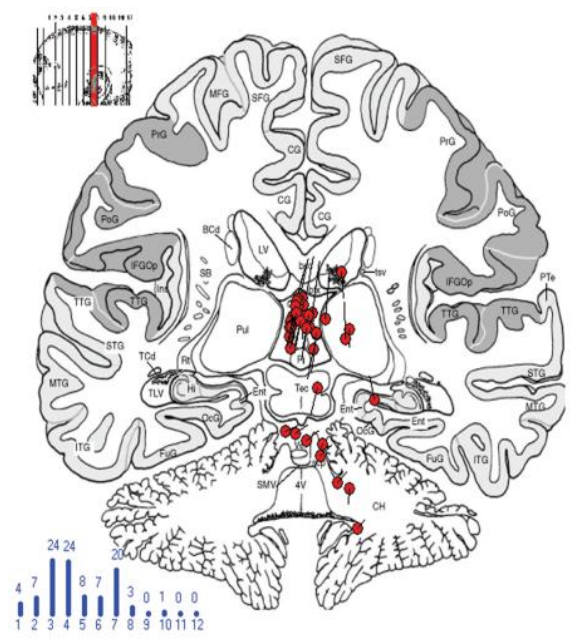

6

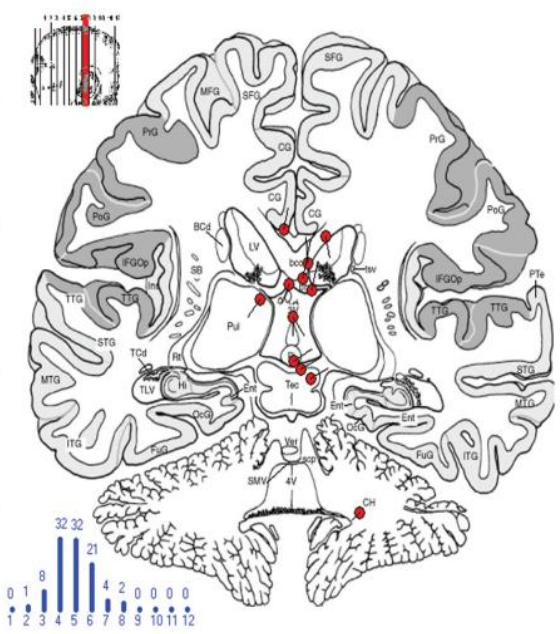

Рис. 2. Подавление доминирующего очага ЭЭДИ в мозжечке у пациента с выраженным сложным моторным тиком с помощью технологии БОС-регуляции (перенос доминирующей активности в другие структуры мозга)

Примечание: а - до регуляции, б - после произвольной регуляции. Остальные обозначения те же, что и на рис. 1.

исходного состояния [10]. Известно, что практически все системы организма находятся в состоянии постоянного неустойчивого равновесия [1], при этом активность структур должна быть в положении некоторой активации [12]. Это позволяет живым системам осуществлять активный поиск оптимальных режимов и реагировать на внешнее воздействие не только повышением, но и понижением своей активности. Если же уровень активации довольно высок - предел его увеличения низок и, наоборот, если активность данной системы низка - предел ее снижения 
невелик. Нейрофизиологической основой этого может быть функциональный резерв системы передачи информации в синаптическом аппарате (истощение медиатора, блокада синапсов или мембранных рецепторов постсинаптической мембраны), активное торможение определенных нервных путей (возвратное или латеральное), истощение энергоресурсов клетки при постоянном воздействии на нее внутренних или внешних факторов, т.е. появление морфофункциональных нарушений. Без учета этих обстоятельств сеансы адаптивного биоуправления могут оказаться не только неэффективными, но даже приводить к еще большей дестабилизации состояния.

\section{ЛИТЕРАТУРА}

1. Бауэр Э. Теоретическая биология. М.-Л. $1935.350 \mathrm{c.}$

2. Бекшаев С.C. Программа: Трехмерная локализация электрических источников головного мозга, порождающих пространственно-временной профиль электроэнцефалограммы. (3DLocEEG). Государственный регистрационный № 2002611116 от 02.07.2002 г.

3. Гнездиикий В.В. Обратная задача ЭЭГ и клиническая электроэнцефалография (картирование и локализация источников электрической активности мозга). М.: МЕДпресс-информ, 2004. 624 с.

4. Гутман А.М. Теория диполя внеклеточного поля // Биофизика. 1968. Т. 13. C. 679-684.

5. Гутман А.М., Шимолюнас А. Теория потенциала ЭЭГ в модели тонких оболочек мозга. IV. Радиальные диполи и их двойные слои в глубине и на поверхности мозга // Биофизика. 1976. Т. 21. С. 898-904.

6. Жадин М.Н. Механизмы возникновения синхронизации биопотенциалов головного мозга отводимых непосредственно от коры. II. Модель зависимых источников // Биофизика. 1972. Т. 17. С. 284-290.

7. Жадин М.Н. Биофизические механизмы формирования электроэнцефалограммы. М.: Наука, 1984. 197 с.

8. Ландау Л.Д., Лившии Е.М. Теорети- ческая физика // Т. 2. Теория поля. М.: Наука, 1967. 460 с.

9. Сороко С.И., Мусуралиев Т.Ж., Комаровер И.Н., Соложенкин В.В. Коррекция нервно-психических дезадаптационных нарушений с помощью метода функционального биоуправления с ЭЭГобратными связями // Физиология человека. Т. 21. № 6. С. 14-28.

10. Сороко С.И., Трубачев В.В. Нейрофизиологические и психофизиологические основы адаптивного биоуправления. СПб.: Политехника-сервис, 2010. 607 с.

11. Фролов А.А., Пономарев В.Н. Влияние покровов мозга на точность локализации источников его биоэлектрической активности в модели однородной неограниченной среды // Журн. высш. нервн. деятельности. 1989. Т. 39. № 1. С. 157-163.

12. Эшби У.Р. Принципы самоорганизации. М. 1966. С. 314-333.

13. Fourment A., Jami L., Calvet J., Scherrer J., Comparison de l'EEG recuelle sur le scalp avec l'activite elementaire des dipoles corticaux radiaires // Electroencephalogr. and Clin. Neurophysiol. 1965. Vol. 19. P. 217-229.

14. Hjorth B. An on-line transformation of EEG scalp potentials into orthogonal source derivations //Electroencephalogr. and Clin. Neurophysiol. 1975. Vol. 39. P. 526530.

15. Mai J.K., Assheuer J., Paxinos G. Atlas of human brain. SanDiego: Academic Press/ 1997. 328 p.

16. Nunez P.L., Srinivasan R., Westdorp A.F., Tucker D.V., Silberstein R.D., Cadusch $P . J$. EEG coherency. Statistics, reference electrode, volume conduction, Laplaqcians, cortical imaging, and interpretation at multiple scales // EEG and Clinical Neurophysiology. 1997. Vol. 103. P. 499-515.

17. Severt T., Longerich U.J., Mai J.K. Topometric and topistic analysis of the humaqn brain. Topometric evalution of histologic brain section // Neurobiologisches Colloquium. Nancy. 1089.

18. Uhlhaas P., Singer W. Neural Synchrony in Brain Disorders: Relevance for Cognitive Dysfunctions and Pathophysiology // Neuron. 2006. Vol. 52. P. 155-168. 